\title{
Stereopsis in end-stage renal disease (ESRD)
}

Article

Accepted Version

Creative Commons: Attribution-Noncommercial-No Derivative Works 4.0

Jones, D. J. W., Harris, J. P., Butler, L. T. and Vaux, E. C. (2017) Stereopsis in end-stage renal disease (ESRD). Physiology \& Behavior, 171. pp. 1-6. ISSN 0031-9384 doi: https://doi.org/10.1016/j.physbeh.2016.12.029 Available at https://centaur.reading.ac.uk/68563/

It is advisable to refer to the publisher's version if you intend to cite from the work. See Guidance on citing.

Published version at: http://www.sciencedirect.com/science/article/pii/S0031938416308356

To link to this article DOI: http://dx.doi.org/10.1016/j.physbeh.2016.12.029

Publisher: Elsevier

All outputs in CentAUR are protected by Intellectual Property Rights law, including copyright law. Copyright and IPR is retained by the creators or other copyright holders. Terms and conditions for use of this material are defined in the End User Agreement.

\section{www.reading.ac.uk/centaur}

\section{CentAUR}

Central Archive at the University of Reading

Reading's research outputs online 
Stereopsis in end-stage renal disease (ESRD)

Daniel J. W. Jones ${ }^{\text {a }}$, John P. Harris ${ }^{b}$, Laurie T. Butler ${ }^{b}$ and Emma C Vaux ${ }^{c}$

a School of Psychology and Clinical Language Sciences, University of Reading Malaysia, Malaysia

${ }^{\mathrm{b}}$ School of Psychology and Clinical Language Sciences, University of Reading, U.K

${ }^{c}$ Department of Renal Medicine, Royal Berkshire NHS Foundation Trust, U.K 


\begin{abstract}
We investigated an effect of end-stage renal disease (ESRD) on the visual system by measuring the ability of 21 patients to perceive depth in the random dot stereograms and circles of the Randot Test. To control for other factors which might influence performance on the tests of stereopsis, patients were compared with healthy controls matched for age, years of education, IQ, and general cognitive ability. Vernier acuity (thought to reflect mainly central processing) and Landolt acuity (more sensitive to retinal and optical abnormalities) were also measured, but the study did not include a formal ophthalmological examination. All controls could perceive depth in random dot stereograms, whereas 9/21 patients could not. Patients who could perceive depth had worse stereoacuity than did their matched controls. The patient group as a whole had worse Vernier and Landolt acuities than the controls. The stereoblind patient subgroup had similar Vernier acuity to the stereoscopic subgroup, but worse Landolt acuity, and were more likely to have peripheral vascular disease. We conclude that ESRD had affected structures both within the eye, and within the visual brain. However, the similarity of Vernier acuity and difference of Landolt acuity in the stereoblind and stereoscopic patient subgroups suggest that the differences in stereoscopic ability arise from abnormalities in the eyes rather than in the brain.
\end{abstract}

\title{
Keywords
}

Stereopsis; end-stage renal disease; impairment; visual acuity; stereo acuity. 


\section{Introduction}

In end-stage renal disease (ESRD), failure of the kidneys leads to changes in the quality of the blood supply to the central nervous system. A variety of cognitive changes has been reported in the illness, including impairments of memory and attention. ${ }^{1-2}$ One might expect that effects of ESRD on the visual brain would produce similar impairments in visual processing, but almost no studies appear to have investigated this. A possible exception is the study by Chiu et $\mathrm{al}^{3}$ in which established haemodialysis (HD) patients were tested on their stereoscopic depth perception, an ability which requires combination in the brain of information from each eye, and which was found to be impaired in many patients. In some chronic illnesses, possible evidence for central impairment of stereopsis has come from imaging studies. Patients with Parkinson's disease who lack stereopsis have reduced grey matter volume in right extrastriate visual cortex compared with patients with stereopsis. ${ }^{4}$ In Alzheimer's disease, maximum responses to stereomotion, as measured with fMRI, occur in different brain regions to those in control participants. ${ }^{5}$ Although it is not clear whether these central effects are the cause or an effect of the changes in stereopsis, changes to visual cortex in ESRD may underlie losses of stereopsis found by Chiu et al. However, there are some problems of interpretation of the results of that study.

1. In the Chiu et al study, participants attempted three parts of the Titmus test, in which they inspected a series of images while wearing polaroid spectacles. In the Housefly sub-test, one is required to touch the wing of a 3-D image of an insect, which after stereoscopic fusion appears above the page on which the two oppositely polarized views are printed. In the Animals subtest, one identifies which of a row of animals is protruding. In the Dots sub-test, one identifies which of four dots forming a cross is protruding. In successive images, the binocular disparity of the protruding dot is gradually decreased, allowing a quantitative measure of stereoacuity. About $30 \%$ of the 
157 patients tested by Chiu et al $^{3}$ failed the Housefly sub-test, and about $58 \%$ and $64 \%$ of those who subsequently attempted the largest disparity versions of the Animals and Dots sub-tests, respectively, failed. A feature of the Titmus test is that the shapes to be seen after binocular combination are also visible in each monocular image. This means that, even when the half-images cannot be stereoscopically fused, the viewer might have some information about the likely position in $3 \mathrm{D}$ space of the feature, as found for stereoscopic images outside Panum's limit by Westheimer and Tanzman. ${ }^{12}$ Another possibility is that visual mechanisms controlling the manual response in the Housefly sub-test might be abnormal, even when those processing the stereogram were functioning normally (though this criticism would not apply to the Animals and the Dots sub-tests). Thus the Titmus test may not give an accurate estimate of losses of stereopsis in ESRD.

2. It is possible that impairment of stereopsis in ESRD results from changes within the eyes rather than in the brain. Ocular abnormalities are common in ESRD, and are often associated with HD. These can include band keratopathy, cataract, macular oedema, retinal haemorrhage and detachment, and optic neuropathy. ${ }^{6}$ The occurrence and nature of another putative symptom (change in intra-ocular pressure) has been questioned, but may be found in some susceptible patients. ${ }^{7}$ Not surprisingly, these anatomical and physiological changes have been associated with various functional losses in ESRD. Luminance thresholds are raised, ${ }^{8}$ visual fields restricted, ${ }^{9}$ and visual acuity and contrast sensitivity reduced. ${ }^{3}$ It is possible that dialysis may contribute to some of these losses. For example, Tomazzoli et al ${ }^{10}$ found reduced visual acuity after dialysis, which could be alleviated with appropriate optical correction, and which they attributed to dehydration of the lens. Since stereoacuity can be impaired by e.g. image blur ${ }^{11}$, it is 
possible that losses of stereopsis in ESRD could be caused by ocular dysfunction of some kind.

3. Chiu et al report that failure on the Housefly test was associated with a low MMSE score and more advanced age, and that many patients refused further testing on the Animals and Dots tests for reasons which included fatigue. Their patients, many of whom may have been frail and cognitively impaired, were compared with published norms, obtained presumably from healthy individuals, so that it is not clear to what extent these other factors may have contributed to their test performance.

We aimed to address these potential difficulties of interpretation as follows.

1. Stereopsis was assessed with two subtests of the Randot Stereotest, namely the Forms and the Circles. The former consists of two arrays of four random dot stereograms, in which easily named shapes (e.g. circle, triangle, the capital letter E) are seen if stereopsis is present. In one array, the binocular disparity of the elements depicting the shape is $500 \mathrm{sec}$, and in the other $250 \mathrm{sec}$, at the recommended viewing distance of 40 $\mathrm{cm}$ for the booklet in which the tests are printed. In each array, one of the stereograms contains no shape, as a check against guessing. In the Circles test, the viewer inspects rows of three circles, one of which protrudes in front of the other two. The disparity of the protruding circle, which is $400 \mathrm{sec}$ on the first row, reduces on successive rows to be $20 \mathrm{sec}$ on the $10^{\text {th }}$ and last row, allowing a quantitative measure of stereoacuity. Thus the Randot Circles sub-test is comparable to the Titmus Dots sub-test. Unlike the Housefly sub-test, however, the Forms sub-test contains no visible monocular clues to the binocularly visible shape, and does not require a precise manual response.

2. With the aim of assessing the relative contribution of retinal and cortical factors in visual losses in ESRD, we carried out two tests of visual acuity from the Freiburg 
Visual Acuity and Vision Test (FrACT) package ${ }^{13}$ : the Landolt C and the Vernier Acuity tests. Chiu et $\mathrm{al}^{3}$ assessed acuity in their patients with the Lighthouse Near Visual Acuity Test (a chart with letters of progressively decreasing size) and found that about $96 \%$ had acuities in the better eye which were worse than the published population norms. The Landolt is sensitive to optical and retinal degradation because it measures spatial resolution, the ability to detect tiny gaps in visual stimuli. We also measured Vernier acuity because the ability to detect an offset between two extended bars is thought to be less dependent on optical and retinal abnormalities and so is more likely to reflect cortical dysfunction. Vernier acuity is finer than the diameter of photoreceptors, and so must depend on processes later in the visual system ${ }^{11}$. That its limit is set by cortical processes is suggested by the finding that, with eccentric viewing, Vernier acuity scales with the cortical magnification factor, unlike spatial resolution ${ }^{14}$, which is measured by the Landolt $\mathrm{C}$ test.

3. Instead of comparing patients with published performance norms, we matched patients with controls of similar age and abilities. In addition to the primary tasks, all participants completed the Mini Mental State Examination (MMSE), ${ }^{15}$ an assessment of general cognitive ability, the Instrumental Activities of Daily Living scale (IADL), ${ }^{16}$ the National Adult Reading Test (NART), ${ }^{17}$ from which pre-morbid IQ can be estimated, and the Geriatric Depression Scale (GDS). ${ }^{18}$ 


\section{Methods}

\section{Participants}

Approval for the study was given by the University Research Ethics Committee and the local National Research Ethics Service (NRES). Twenty-one patients, ranging from 37 to 88 years of age (mean age: 67.7, SD: 14.45) were recruited from the renal unit at the Royal Berkshire Hospital (RBH) in Reading, Berkshire, UK. These ESRD patients received HD three times per week for 3 to 5 hours per treatment and had been receiving HD treatment for a minimum of 90 days prior to testing, with a Kt/v > 1.4 (dialysis adequacy). Informed consent was obtained by a nephrologist independent of the study. Details of comorbid conditions and other relevant history were obtained from medical records. Patients were excluded from the study if they had any prior history of ophthalmological or neurological illness. Thus, three potential participants were excluded: one was blind in one eye, the second had had surgical correction of a squint, and the third had had a retinal detachment. It is possible that some of the patients and controls who were tested had other visual problems of which they were unaware or unwilling to report. Testing was conducted in a quiet office in the RBH.

Twenty-one healthy control participants (mean age: 67.3 years, SD: 14.2) were recruited from a research volunteer panel maintained by the Department of Psychology at the University of Reading. Potential participants were excluded if they had a history of renal, ophthalmological, or neurological illness. Thus, one was excluded because they reported very poor vision in one eye, from a young age. Control participants were individually matched to the patients for age, sex, and education level. They were tested in a quiet room within the Department of Psychology, after informed consent had been obtained, and were reimbursed for their travelling expenses. 
Each patient was tested immediately before a dialysis session (and so at least two days since the previous dialysis). Their matched control was tested at approximately the same time of day.

\section{Test Materials and Procedure}

Visual tests

Both testing rooms were lit by conventional fluorescent lighting of about 500 lux. Thus all stimuli were viewed in conditions which were comfortably within the photopic range. FrACT stimuli were presented on a Toshiba 17.3" LCD screen laptop computer, and viewed binocularly. The participant was seated in a comfortable chair, at a viewing distance of $180 \mathrm{~cm}$ from the screen. In the Landolt $C$ test, a series of 24 presentations was made, in each of which the gap in a circle can be in one of 8 positions. If the participant correctly identifies the location of the gap it is reduced on the next presentation, or, if an error is made, it is maintained or reduced on the next presentation, according to an adaptive staircase procedure which homes in on a chosen level of performance (62.5\% at a $25 \%$ guessing rate). On the initial presentation, the outer diameter of the $\mathrm{C}$ was $48 \mathrm{~min}$ arc, and the gaps width was 9.5 min arc. In the Vernier acuity task, a series of 42 vertical Verniers is presented, with the lower line offset to the right or left of the upper line, and the participant judges the direction of the offset. Each bar of the Vernier stimulus was $14 \operatorname{arc}$ min long x 2 arc min wide. The initial offset was 2 arc min. As in the Landolt $\mathrm{C}$ procedure, the offset is varied according to the participant's performance on successive presentations, until some criterion level of performance is reached.

For the Randot tests, which followed the acuity tests, the seated participant wore polaroid spectacles and held the booklet at a distance of aproximately $40 \mathrm{~cm}$. The nature of the two subtests is described above in a comparison with the sub-tests of the Titmus test. For the 
Forms test, the participant was required to point to and describe the shapes in each array of stereograms, first those with $500 \mathrm{sec}$ disparity, then those with $250 \mathrm{sec}$ disparity. Following this, they were required to identify the location of the protruding circle in the Circles test, beginning with the largest disparity. Stereoacuity was taken to be the disparity before the stimulus at which the participant made an error or reported that they could not differentiate between the depths of the three circles. In the latter case, they were not forced to guess. Participants wore their usual optical correction for the viewing distances in the visual tests.

\section{Neuropsychological tests}

\section{Mini Mental State Exam (MMSE)}

The $\mathrm{MMSE}^{15}$ is a short questionnaire designed to screen for cognitive impairment. The 30 questions assess memory, orientation to time, orientation to place, attention and language. A score of 25-30 indicates normal cognition, 21-24 mild, 10-20 moderate and $<9$ severe impairment.

\section{National Adult Reading Test (NART)}

The NART ${ }^{16}$ assesses pre-morbid intelligence in English-speaking individuals. Fifty words, whose reading difficulty varies, are shown on a single sheet, and have to be read aloud. Overall, verbal and performance IQ can be estimated from the number of words correctly pronounced.

Instrumental Activities of Daily Living scale (IADL)

The IADL ${ }^{17}$ assesses an individual's independence and how they deal with the demands of everyday life. Eight different domains are assessed, including food preparation, driving, and shopping; the scale ranges from 0 (low independence) to 8 (full independence). 
Geriatric Depression Scale (GDS)

The GDS ${ }^{18}$ is a 15 -item screening tool for depression in the elderly. It is short and easy to administer using a number of questions about mood and anxiety to estimate a score of depression. A score of 0-4 is considered normal, 5-9 is mild depression and 10-15 is more severe depression.

\section{Results}

Demographic and medical information

Some characteristics of the patient and control groups are shown in Table 1. It may be seen that the groups were well matched for age, years of education, overall (pre-morbid) IQ (NART) and general cognitive ability (MMSE). From their GDS scores, the patients showed significantly more signs of depression than the controls, though as a group their symptoms were mild (a score of 4 or less is considered normal). The patients were also mildly impaired in activities of daily living (the maximum score, achieved by all controls, is 8 ). Table 2 shows some medical characteristics of the patient group, including duration of dialysis, cause of ESRD, and co-morbidities. 
Table 1. Demographic information for patient and control groups.

\begin{tabular}{|c|c|c|c|c|c|c|}
\hline & \multicolumn{4}{|c|}{ Group } & \multirow[b]{3}{*}{$t$} & \multirow[b]{3}{*}{$p$-value } \\
\hline & \multicolumn{2}{|c|}{ Patient $(\mathrm{N}=21)$} & \multicolumn{2}{|c|}{ Control $(\mathrm{N}=21)$} & & \\
\hline & Mean & SD & Mean & SD & & \\
\hline Age (yr.) & 67.7 & 14.5 & 67.3 & 14.2 & 0.099 & 0.921 \\
\hline Education (yr.) & 11.9 & 2.7 & 12.2 & 2.6 & -0.353 & 0.726 \\
\hline NART Overall IQ & 109.4 & 25.9 & 111.2 & 26.9 & -0.219 & 0.828 \\
\hline GDS & 4.2 & 3.3 & 1.8 & 1.5 & 2.897 & .006 \\
\hline MMSE & 27.7 & 2.1 & 28.5 & 1.0 & -1.545 & .131 \\
\hline IADL & 6.6 & 1.5 & 8.0 & 0.0 & -3.924 & $<.001$ \\
\hline $\mathrm{M} / \mathrm{F}$ & $13 / 8$ & - & $13 / 8$ & - & - & - \\
\hline
\end{tabular}


Table 2. Medical characteristics of ESRD patients.

HD Patients $(n=21)$

Characteristic

No. of Patients Percentage

Age (yr.)

$<55$

4

19.1

$55-64$

5

23.8

$65-74$

3

14.3

$75-85$

8

38.1

$>85$

1

4.8

Dialysis Duration (months)

$0-12$

3

14.3

13-24

5

23.8

$>24$

13

61.9

Cause of ESRD

Type 2 diabetes mellitus

3

14.3

Type 1 diabetes mellitus

1

4.8

Adult polycystic kidney disease

2

9.5

Chronic kidney disease (unknown cause) 4

19.0

Obstructive uropathy

9.5

Glomerulonephritis

2

23.8

Vasculitis

5

9.5

Hypertensive/renovascular disease

4.8

Surgical loss

4.8

Comorbid Conditions

Peripheral vascular disease

7

33.3

Diabetes

Hypertension

15

71.4

Stroke

3

14.3

Myocardial infarction

1

4.8 
Vernier and Landolt acuities

Mean Landolt and Vernier acuities in the patient and control groups are shown in Figure 1. Independent t-tests revealed that patients had significantly lower acuity than controls on the Landolt $C$ test $(t(39)=3.399, p=0.002)$, and also (after log transformation of the scores to equate the variances) on the Vernier task $(t(37)=3.037, p=.004)$. Due to testing fatigue, one control did not complete the Landolt task, and two patients and one control did not complete the Vernier test.

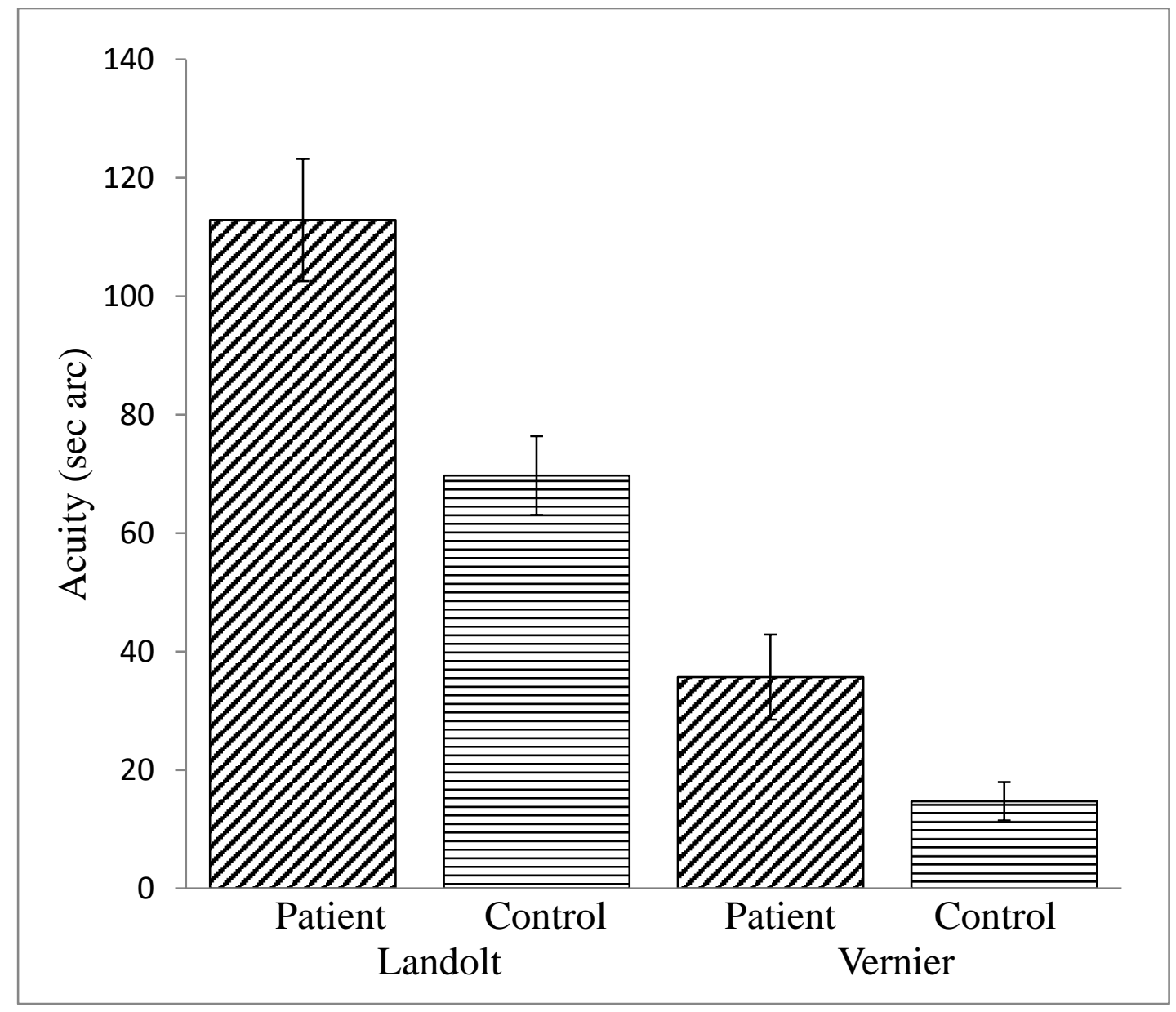

Figure 1. Mean Landolt C and Vernier acuities for the ESRD and control groups. Error bars $= \pm 1$ S.E. 


\section{Stereopsis}

9 of the 21 patients (42.9\%) could not perceive the figures in the Randot Forms subtest, whereas all the controls could do so (a difference which was significant (Fisher's Exact $p=$ 0.0007)). None of these 9 patients could perform the Circles test, even at the largest disparity. The mean stereoacuity from the Circles tests of the 12 patients who could identify the figures in the Forms test was $139.17 \mathrm{sec}$ arc $(\mathrm{SE}=39.68)$, that of their matched controls $53.33 \mathrm{sec}$ $\operatorname{arc}(\mathrm{SE}=15.8)$. This difference was significant $(t(22)=2.124, p=0.045$, after $\log$ transformation of the scores to equate the variances).

Figure 2 shows Landolt and Vernier acuities for the stereoblind and stereoscopic sub-groups of patients. Landolt acuity was worse in the stereoblind group, with the difference from

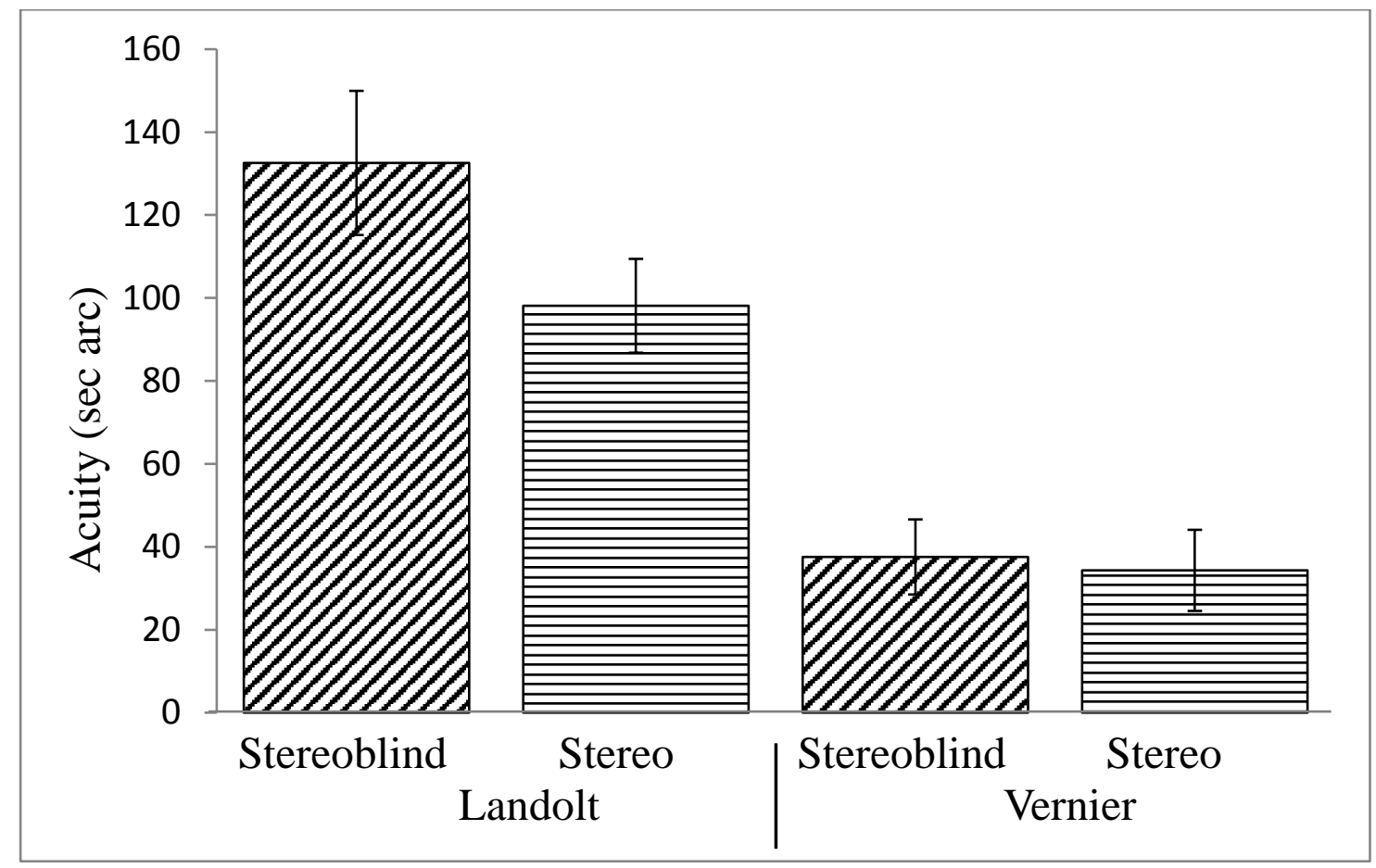

Figure 2. Mean visual acuities in stereoblind patients with ESRD and those with stereopsis. Error bars $= \pm 1$ S.E. 
the stereoscopic subgroups approaching significance on a parametric test $(t(19)=1.732, p=$ 0.09). On a test of medians, the sub-groups were significantly different (Fisher's Exact $p=0.03$ ). Vernier acuities were almost identical in the two sub-groups, and the difference between them was not significant, either with a parametric $(t(19)=0.144, p=0.89)$ or a nonparametric test (Fisher's Exact $p=0.99$ ).

Other characteristics of the stereoblind and stereoscopic subgroup are shown in Table 3. None of the differences in years of education, or of scores on the NART, GDS, MMSE or IADL were significant on a parametric test, nor on a comparison of medians for the smallest differences (Fisher's Exact $p$ for age $=0.09$, for duration of dialysis $=0.67$, for years of education $=0.99$, and for $\mathrm{IADL}=0.08$ ). Although the stereoblind group tended to be older, and to have spent more time on dialysis, the differences between the groups were not significant. There were no significant differences between the groups in the incidence of hypertension, stroke, myocardial infarction or diabetes (though it is possible that the two diabetics in the stereoblind group had undiagnosed retinopathy). However, peripheral vascular disease was significantly more common in the stereoblind group (55.5\%) than in the stereoscopic group $(8.3 \%)$ - Fisher's Exact $p=0.046$

\section{Correlations}

In the control group, none of the correlations between the three tests of acuity was significant (largest $r=0.312$, smallest $p=0.18, n=21$ ). The only significant correlation between an acuity and a demographic or neuropsychological measure was between Landolt acuity and age $(r=0.655, p=0.002, n=21)$; older people having worse acuity.

The correlation between Landolt and Vernier acuities in the patients did not reach significance $(r=0.375, p=0.094 ; n=19)$. Landolt acuity in patients correlated significantly with age ( $r=0.642, p=0.002, n=20$ - older people were worse). Both acuities correlated 
significantly with activities of daily living (Landolt: $r=-0.497, p=0.022, n=20$; Vernier: $r=-$ $0.452, p=0.040, n=19)$ : in both cases, worse acuity was associated with greater impairment in tasks of everyday living. In the patients with stereopsis, stereoacuity correlated positively and significantly with Landolt acuity $(r=0.698, p=0.012 ; n=12)$, but not with Vernier acuity $(r=0.351, p=0.264)$. Patient stereoacuity did not correlate with age or years of education, or with scores on the NART, GDS, MMSE or IADL (largest $r=0.542$, smallest $p=0.069, n=12$ ).

Table 3. Characteristics of ESRD stereoblind and stereoscopic groups

\section{Group}

\section{Stereoblind (N = 9) $\quad$ Stereo $(\mathbf{N}=12)$}

\begin{tabular}{llllll}
\hline Mean & SD & Mean & SD & $t$ & $p$-value
\end{tabular}

\begin{tabular}{lllllll}
\hline Age (yr.) & 72.7 & 14.0 & 64.0 & 14.2 & 1.392 & 0.180 \\
Dialysis duration (mo) & 53.3 & 57.2 & 33.4 & 19.1 & 1.42 & 0.171 \\
Education (yr.) & 10.7 & 1.7 & 12.8 & 3.1 & -1.82 & 0.085 \\
NART Overall IQ & 109.4 & 25.9 & 111.2 & 26.9 & -0.219 & 0.828 \\
GDS & 4.3 & 2.8 & 4.1 & 3.8 & 0.167 & 0.889 \\
MMSE & 27.7 & 2.3 & 27.7 & 1.9 & -0.000 & 1 \\
IADL & 6.3 & 1.2 & 6.8 & 1.7 & -0.731 & 0.473 \\
\hline M / F & $5 / 4$ & - & $7 / 5$ & - & - & - \\
\hline
\end{tabular}




\section{Discussion}

In all three tests of acuity, ESRD patients performed worse than controls. Thus, our findings with the Landolt $\mathrm{C}$ and Randot tests confirm those of Chiu et $\mathrm{al}^{3}$ with the Lighthouse Near Acuity chart and the Titmus test. As our patients were matched with healthy controls for age, IQ and general cognitive ability, we can be more confident that our findings reflect a visual loss rather than some other difficulty in carrying out the tests.

A striking feature of the data was the lack of any measurable stereoscopic ability in more than $40 \%$ of our patients. That this occurred with random dot stereograms, in the absence of monocular cues, suggests a genuine difficulty in binocular combination. The remaining patients, who could perceive depth in random dot stereograms, nevertheless had worse stereoacuity than did their matched controls. The stereoblind and stereoscopic ESRD subgroups could not be distinguished on any of our demographic or neuropsychological measures (see Table 3). The only significant difference in co-morbidity was a significantly higher incidence of peripheral vascular disease in the stereoblind group. The two groups had almost identical Vernier acuity. The only other apparent distinguishing feature was a difference in their Landolt acuity (see Figure 2).

What do the results tell us about the effects of ESRD on the visual system? The worse Vernier acuity in the patient group as a whole suggests some impairment in cortical processing, perhaps a reduction in orientation discrimination ${ }^{19}$. However, the similar performance of the stereoblind and stereoscopic subgroups on the Vernier acuity test suggests a similarity in at least one aspect of cortical processing, despite the differences in binocular combination. In contrast, our data from the Landolt test, showing greater impairment in the stereoblind subgroup, appear to be consistent with differences within the eyeball, either optical or retinal, between the two patient subgroups. For example, Quaid et $\mathrm{al}^{20}$ showed that 
blurring with positive lenses or with contact lenses which simulate cataracts had a greater effect on letter than on Vernier acuity. In their study, blurring had little effect on Vernier acuity below two dioptres. Blurring impairs the perception of random dot stereograms ${ }^{21}$ and stereoacuity. ${ }^{22}$ The effects of contrast reduction are greater on stereoacuity than on Vernier acuity. ${ }^{20}$ Thus the differences we found between the stereoblind and stereoscopic subgroups could result from some optical degradation (perhaps refractive error, cataract, and/or deposits in the cornea) which was large enough to produce a differential effect on Landolt acuity and stereoacuity, but not large enough to affect Vernier acuity differentially. This notion is also supported by the correlation between Landolt acuity and stereoacuity in the stereoscopic patient subgroup. Although uncorrected refractive error is not uncommon in older people (see studies summarised by Shickle and Griffin ${ }^{23}$ ), it seems unlikely that this alone could account for the differences between the patient and control groups in our study. For example, any effects on refraction through dehydration of the lens caused by dialysis in the patient group (as found by Tomazzoli et al, ${ }^{10}$ ) would have dissipated after two days, and one might expect any residual refractive errors to be spread equally over the patient and control groups.

It is also possible that there might be additional differences in retinal processing between the patient subgroups; of the four diabetic patients, two had stereopsis and two were stereoblind. It is possible that the latter two patients had diabetic retinopathy, though this was not mentioned in their medical records. The stereoblind subgroup had a higher incidence of peripheral vascular disease, which in turn might be associated with greater impairment of retinal processing. Such vascular changes in the retina have been measured in ESRD ${ }^{24}$. This may affect Vernier acuity less than the other acuities because the positions of the extended bars of the Vernier stimuli could be perceived accurately despite any tiny scotomata caused by local vascular failures. Thus we conclude that present evidence does not support the idea that the losses of stereopsis in ESRD found by Chiu et al and in this study reflect changes in 
the cortical mechanisms of binocular combination. It is also possible that errors in oculomotor control may contribute to the stereoscopic losses found here, since it is known that vergence error and vergence variability may elevate stereoscopic thresholds in normal participants ${ }^{25}$. However, such errors may not be a complete explanation for stereoblindness in the patient group, since image motion analogous to that used in the study of vergence variability also impairs Vernier acuity, ${ }^{26}$ and this was similar in the stereoblind and stereoscopic patient sub-groups.

The correlation between age and Landolt acuity in both the patient and the control groups is predictable from previous work. It is likely that there is a neural as well as an optical component in the visual losses associated with ageing. With direct viewing of gratings, contrast sensitivity is worse in elderly than in young adults ${ }^{27}$, the losses rising from about 0.2 $\log$ units at $2 \mathrm{c}$ deg- 1 to about $0.5 \log$ units at $16 \mathrm{c} \mathrm{deg}^{-1}$. Burton et $\mathrm{a}^{28}$ later showed that when gratings are produced on the retina with a technique (laser interferometry) which bypasses the eye's optics, sensitivity in the elderly is about $0.1 \log$ units lower at all spatial frequencies tested. They concluded that their data provide evidence for neural as well as optical losses with ageing. The possible optical contributions include increasing opacity of the lens and cornea as well as a decrease in pupil diameter. The neural losses are likely to be mostly cortical. This Ahmad and Spear ${ }^{29}$ and Spear et $\mathrm{al}^{30}$ found only minor changes in LGN responses in elderly monkeys, whereas V1 cells have lower preferred spatial frequencies and reduced contrast sensitivities ${ }^{31}$.

The reduced acuities found in the patient group may have some practical consequences. In the patients (but not the controls), both Landolt and Vernier acuity correlated with IADL scores, but stereoacuity did not. Nor were IADL scores worse in the stereoblind than in the stereoscopic patients (see Table 3). However, the stereoblind patients may well be worse on some tasks, such as threading a needle, which are not covered by the IADL questionnaire, 
though for many tasks, such as shopping or financial management, the ability to resolve fine spatial detail may be more important. It seems likely that many ESRD patients will have difficulty in reading small print, as often found on the containers of prescribed drugs, and perhaps be more likely to fall than the healthy elderly ${ }^{32}$.

\section{Limitations of the study}

We did not fix viewing distances precisely with chin-rest and brow-bar in the visual tests, therefore it is likely that there will have been small individual variations. However, there is no reason to suppose that viewing distances would have been systematically shorter in one of the groups. Rather, any effect would be an increase in random variation in patients and controls. A potentially important limitation is that of sample size: the stereoblind and stereoscopic patient groups contained 9 and 12 participants respectively. Thus some of the trends towards differences (in age, or months on dialysis) or correlations with neuropsychological measures which we found might become significant with larger numbers of participants. However, this would not affect our main conclusions. Finally, the absence of an ophthalmological examination of the participants means that our conclusions must be viewed with caution. Such an examination would be an important addition to future studies of stereopsis in ESRD.

\section{Conclusions}

In summary, we have confirmed earlier findings that stereopsis and visual acuity are impaired in ESRD, and found that Vernier acuity is also worse. By comparing patients with matched controls, we can rule out years of education, IQ, and general cognitive state as contributing to patients' difficulties on our tests. More than $40 \%$ of the patients were stereoblind, as assessed by the Randot Forms test, and those with stereopsis had lower stereoacuity than their matched controls. Vernier acuity was similar in the stereoscopic and stereoblind sub-groups, 
but Landolt $\mathrm{C}$ acuity was worse in the stereoblind. We suggest that the impairments of stereopsis in ESRD result from changes in the eye, perhaps retinal as well as optical. In the patients, lower Vernier and Landolt acuities correlated with lower IADL scores, suggesting that inability to resolve fine detail in ESRD may be affecting the tasks of everyday life. Further explorations need to be made, however, our data suggest that visual testing would be a useful adjunct to the treatment of ESRD.

\section{Acknowledgements}

We thank all our participants for taking part in the study, and the staff of the RBH renal wards for their help. This study was funded by the Economic and Social Research Council (ESRC) CASE studentship (grant no. ES/H039821/1). 


\section{References}

1. Elias, M. F., Elias, P. K., Seliger, S. L., Narsipur, S. S., Dore, G. A., \& Robbins, M. A. (2009). Chronic kidney disease, creatinine and cognitive functioning. Nephrology Dialysis Transplantation, 24, 2446-2452.

2. Jones DJW, Harris JP, Vaux E, Hadid R, Kean R, Butler LT (2015) The nature of impairments of memory in patients with End Stage Renal Disease (ESRD). Physiology and Behavior 147, 324-333

3. Chiu E, Markowitz SN, Cook WL \& Jassal SV. Visual impairment in elderly patients receiving long-term haemodialysis. Am J Kidney Dis 2008; 52(6): 1131-1138.

4. Koh SB, Suh SI, Kim SH, Kim JH (2013) Stereopsis and extrastriate cortical atrophy in Parkinson's disease: a voxel-based morphometric study. Neuroreport24(5), 229232.

5. Thiyagesh SN, Farrow TFD, Parks RW, Accosta-Mesa H, Young C, Wilkinson ID, Hunter MD, Woodruff PWR ( 2009) The neural basis of visuospatial perception in Alzheimer's disease and healthy elderly comparison subjects: an fMRI study. Psychiatry Research: Neuroimaging 172, 109-116

6. Mullaem G \& Rosner MH. Ocular problems in the patient with End-Stage Renal Disease. Semin Dial 2012; 25(4): 403-407.

7. Levy J, Tovbin D, Lifshitz T, Zlotnik M \& Tessler J. Intraocular pressure during haemeodialysis: a review. Eye 2005; 19: 1249-1256.

8. Pahor D. Retinal light sensitivity in haemodialysis patients. Eye 2003; 17: 177-182.

9. Pelit A, Zumrutdal A \& Akova Y. The effect of haemodialysis on visual field test in patients with chronic renal failure. Curr Eye Res 2003; 26(5): 303-306.

10. Tomazzoli L, de Natale R, Lupo A \& Parolini B. Visual acuity disturbances in chronic renal failure. Ophthalmologica 2000; 214: 403-405. 
11. Westheimer G. The spatial sense of the eye: Proctor lecture. Invest Ophthalmol Vis Sci 1979; 18(9): 893-912.

12. Westheimer G \& Tanzman IJ. Qualitative depth localization with diplopic images. J Opt Soc Am 1956; 46(2): 116-117.

13. Bach M. The "Freiburg Visual Acuity Test" - Automatic measurement of visual acuity. Optom Vis Sci 1996; 73: 49-53, http://michaelbach.de/fract/

14. Levi DM, Klein SA, Aitsebaomo AP (1985) Vernier acuity, crowding and cortical magnification. Vision Research 25(7), 963-977

15. Folstein MF, Folstein SE, \& McHugh PR. "Mini-Mental State": A practical method for grading the cognitive state of patients for the clinician. J Psychiatr Res 1975; 12 : 189-198

16. Nelson HE, \& Willeson JR. National Adult Reading Test (2nd ed.). Windsor, UK: NFER-Nelson, 1991.

17. Lawton MP, \& Brody EM. Assessment of older people: Self-maintaining and instrumental activities of daily living. Gerontologist 1969; 9: 179-186.

18. Yesavage JA, Brink TL, Rose TL et al. Development and validation of a geriatric depression screening scale: A preliminary report. J Psychiatr Res 1983; 17: 37-49.

19. Fahle M, Harris JP (1998) The use of different orientation cues in vernier acuity. Perception \& Psychophysics 60(3), 405-426

20. Quaid P, Stonier C, Cox MJ. Potential vision testing - the relationship between visual acuity and Vernier acuity in the presence of simulated cataracts. Ophthalmic Physiol Opt 2002; 22: 469-481.

21. Odell NV, Hatt SR, Leske DA, Adams WE \& Holmes JM. The effect of induced monocular blur on measures of stereoacuity. J AAPOS 2009; 13(2): 135-141. 
22. Westheimer G \& Pettet MW (1990) Contrast and duration of exposure differentially affect vernier and stereoscopic acuity. Proc R Soc Lond B Biol Sci 1990; 241(1300): $42-46$.

23. Shickle D, Griffin M (2014) Ophthalmic and Physiological Optics 34, 38-45. doi: 10.111/opo. 12100

24. Yaffe K, Ackerson L, Hoang TD, Go AS, Maguire MG, Ying G-S, Daniel E, Bazzano LA, Coleman M, Cohen DL, Kusek JW, Ojo A, Selger S, Xie D, Grunwald JE (2013) Retinopathy and cognitive impairment in adults with CKD. American Journal of Kidney Disease 61(2), 2019-227

25. Ukwade MT, Bedell HE, Harwerth RS (2003) Stereopsis is perturbed by vergence error. Vision Research 43: 181-193

26. Bedell HE, Ukwade MT (1997) Sensory deficits in idiopathic congenital nystagmus. In: Basic and Clinical Applications of Vision Science (V Lakshiminarayanan, ed). Kluwer Academic Publishers, Dordrecht, pp. 251-255.

27. Owsley C, Sekuler R, Siemsen D (1983) Contrast sensitivity throughout adulthood. Vision Research 23(7): 689-699

28. Burton KB, Owsley C, Sloane ME (1993) Aging and neural spatial contrast sensitivity: photopic vision. Vision Research 33(7): 939-946

29. Ahmad A, Spear PD (1993) Effects of aging on the size, density, and number of rhesus monkey lateral geniculate neurons. Journal of Comparative Neurology 334: $631-643$

30. Spear PD, Moore RJ, Kim CBY, Xue J-T, Tumosa N (1994) Effects of aging on the primate visual system: spatial and temporal processing by lateral geniculate neurons in young adult and old rhesus monkeys. Journal of Physiology 72(1): 402-420 
31. Zhang J, Wang X, Wang Y, Fu Y, Liang Y, Ma Y, Leventhal AG (2008) Spatial and temporal sensitivity degradation of primary visual cortical cells in senescent rhesus monkeys. European Journal of Neuroscience 28: 201-207

32. Lord S R, Dayhew J. (2001), Visual Risk Factors for Falls in Older People. Journal of the American Geriatrics Society 49, 508-515. doi:10.1046/j.15325415.2001.49107.x 